\title{
Estoque e descarte de medicamentos no domicílio: uma revisão sistemática
}

\author{
Storage and disposal of pharmaceuticals at home: a systematic \\ review
}

\author{
Viviane Macedo Constantino (https://orcid.org/0000-0001-6716-3574) ${ }^{1}$ \\ Brisa Maria Fregonesi (https://orcid.org/0000-0002-6800-8629) ${ }^{1}$ \\ Karina Aparecida de Abreu Tonani (https://orcid.org/0000-0002-3723-819X) ${ }^{1}$ \\ Guilherme Sgobbi Zagui (https://orcid.org/0000-0002-6104-4360) ${ }^{1}$ \\ Ana Paula Contiero Toninato (https://orcid.org/0000-0002-7251-6423) ${ }^{1}$ \\ Eliana Roldão dos Santos Nonose (https://orcid.org/0000-0002-1998-1684) ${ }^{1}$ \\ Luciana Aparecida Fabriz (http://orcid.org/0000-0001-7633-0127) ${ }^{1}$ \\ Susana Inés Segura-Muñoz (https://orcid.org/0000-0002-6720-8231) ${ }^{1}$
}

${ }^{1}$ Escola de Enfermagem de Ribeirão Preto, Universidade de São Paulo. Av.

Bandeirantes 3900, Campus Universitário. 14040-900

Ribeirão Preto SP Brasil. susis@eerp.usp.br

\begin{abstract}
This study's objective was to analyze Brazilian and international scientific evidence on the reasons people store medication at home and the disposal methods used. This systematic literature review includes studies published from 2001 to 2016 and indexed in PubMed, Lilacs and Elsevier's Scopus Database, using the following key words: Disposal of medicines in household, Disposal of drugs expired, Residential expired drugs, Management of expired medications in household. Thirty-three studies were selected after applying inclusion and exclusion criteria. The studies reveal that the main reasons people keep a stock of medicines at home include the possibility of future use, leftovers from previous treatments, and self-medication. Inappropriate disposal methods prevail, such as throwing medicines in the household trash or flushing them into the sewer. Some countries have adopted programs to educate on proper storage and disposal of medicines, though only one study describes a national program guiding these practices that is free of charge. Evidence shows there is a need to implement public policies intended to provide guidance on the proper storage and disposal of medicines at home. Key words Medication storage, Solid waste collection, Collection routes, Housing
\end{abstract}

Resumo Este estudo teve como objetivo analisar a evidência científica nacional e internacional existente sobre o motivo do estoque e a forma de descarte dos medicamentos no domicílio. Realizou-se uma revisão sistemática da literatura de 2001 a 2016 nas bases de dados PubMed, Lilacs e Elsevier's Scopus Database, utilizando os seguintes unitermos: "Disposal of medicines in household", "Disposal of expired drugs", "Residential expired drugs", "Management of expired medications in household". Foram selecionados 33 estudos originais após aplicação dos critérios de inclusão e exclusão. Os estudos analisados demonstraram que os principais motivos de estoque no domicílio foram: automedicação; guarda para uso futuro e sobras de tratamentos anteriores. E quanto ao descarte dos medicamentos, prevalecem práticas inadequadas, como descarte no lixo comum e na rede de esgoto. Alguns países têm adotado programas de orientação sobre o armazenamento e o descarte correto, no entanto, foi apenas encontrado um estudo que descreve um programa nacional gratuito, para orientar essas práticas. A partir das evidências cientificas verificou-se a importância do incentivo para a elaboração e a implementação de políticas públicas voltadas para o uso e descarte adequado de medicamentos no domicílio.

Palavras-chave Armazenamento de medicamentos, Coleta de resíduos sólidos, Rotas de coletas, Habitação 


\section{Introdução}

O desenvolvimento tecnológico possibilitou diversos avanços no campo das ciências, especialmente na área da saúde. Nesse contexto, destacam-se os avanços das ciências farmacêuticas e da medicina, as quais ampliaram as possibilidades e quantidade de medicamentos disponíveis para a comercialização e consumo ${ }^{1}$.

Os medicamentos são eficazes no tratamento de inúmeras doenças, sendo fundamentais no cuidado da saúde da população. No entanto, estimativas da Organização Mundial da Saúde (OMS) apontam que $50 \%$ de todos os medicamentos são prescritos, dispensados ou usados de maneira incorreta ${ }^{2}$. Ainda nesse sentindo, de acordo com o Centers for Disease Control and Prevention (CDC), todos os anos aproximadamente 80 milhões de antibióticos são prescritos inadequadamente ${ }^{3}$.

Em 2016, a Organização Pan-Americana de Saúde emitiu novas diretrizes sobre o uso racional de medicamentos, contemplando a orientação para o armazenamento em domicílios". Embora essas diretrizes proponham a utilização racional dos produtos terapêuticos, o armazenamento de medicamentos nos domicílios ainda é uma prática comum. Em diversos países do mundo, a cultura de manter "mini farmácias caseiras" (estoque domiciliar de medicamentos), propicia o consumo irracional de medicamentos favorecendo a automedicação, a ocorrência de acidentes e o acúmulo de diversos produtos químicos no interior do domicílio ${ }^{5,6}$.

No Brasil está vigente a Política Nacional de Medicamentos (2001) que têm como objetivo garantir o acesso da população aos medicamentos essenciais, de qualidade, promovendo seu uso racional ${ }^{7}$. Para a definição dos critérios sobre prescrição, dispensação, controle, embalagem e rotulagem de medicamentos foram instituídas as resoluções RDC No 20/2011 e RDC 68/2014 pela Agência Nacional de Vigilância Sanitária (Anvi$\mathrm{sa}^{8,9}$. Apesar das normativas quanto ao uso racional, controle e dispensação de medicamentos, o acúmulo dos mesmos e o seu descarte inadequado representam ainda uma preocupação para a saúde pública e ambienta ${ }^{10-12}$.

Diversos estudos nacionais e internacionais apontam a presença de fármacos e princípios ativos contaminando solos e águas ${ }^{13,14}$. Porém, ainda existem lacunas na compreensão dos motivos que levam a população a estocar medicamentos e na identificação das rotas comumente utilizadas para o descarte ${ }^{1,15,16}$. Nesse contexto, o objetivo do presente estudo foi analisar a evidência científica nacional e internacional existente sobre os motivos do estoque de medicamentos no domicílio e as usuais formas de descarte.

\section{Metodologia}

Trata-se de uma Revisão Sistemática da Literatura (RSL), utilizando o percurso metodológico descrito por Olsen ${ }^{17}$ e adaptado por Ferreira et al. ${ }^{18}$, o qual comtempla: a definição do problema de pesquisa, o desenho do teste de relevância com o estabelecimento dos critérios de inclusão e exclusão, a escolha das bases de dados e unitermos a serem utilizados, com o subsequente processo de seleção dos estudos.

Para nortear esta revisão sistemática, foi elaborada a seguinte questão: Quais as evidências cientificas, nacionais e internacionais sobre os motivos do estoque de medicamentos no domicílio e as usuais formas de descarte?

O desenho do Teste de Relevância (TR ${ }^{17,18}$ contemplou os seguintes critérios de inclusão: a) Estudos que tratassem do estoque e ou descarte de medicamentos; b) Estudos que abordassem medicamentos no domicílio; c) Estudos referentes às sobras de medicamentos vencidos ou não; d) Estudos publicados no período de 2001 a 2016; e) Estudos nos idiomas: Inglês, Português ou Espanhol. E foram excluídos: revisões, relatos de caso, comunicações, monografias e resumos.

A busca pelos artigos fora realizada em junho de 2017 nas seguintes bases de dados: Literatura Latino-Americana e do Caribe em Ciências da Saúde (Lilacs), U.S. National Library of Medicine and the National Institutes Health (PubMed) e Elsevier's Scopus Database, utilizando os seguintes unitermos: Disposal of medicines in household; Disposal of expired drugs; Residential expired drugs; Management of expired medications in household.

A pesquisa nas bases de dados, a aplicação do TR e a seleção dos artigos na íntegra foram realizadas por dois pesquisadores de forma independente, com a finalidade de verificar a objetividade do método, sendo que nos casos de divergências sobre a inclusão um terceiro pesquisador foi consultado.

De acordo com o fluxograma da Figura 1, na consulta às bases de dados foram encontrados 268 artigos dos quais 47 foram excluídos, pois apresentavam duplicidade. Foi então realizada a primeira aplicação do TR nos resumos, permanecendo 63 artigos que foram lidos na íntegra. 


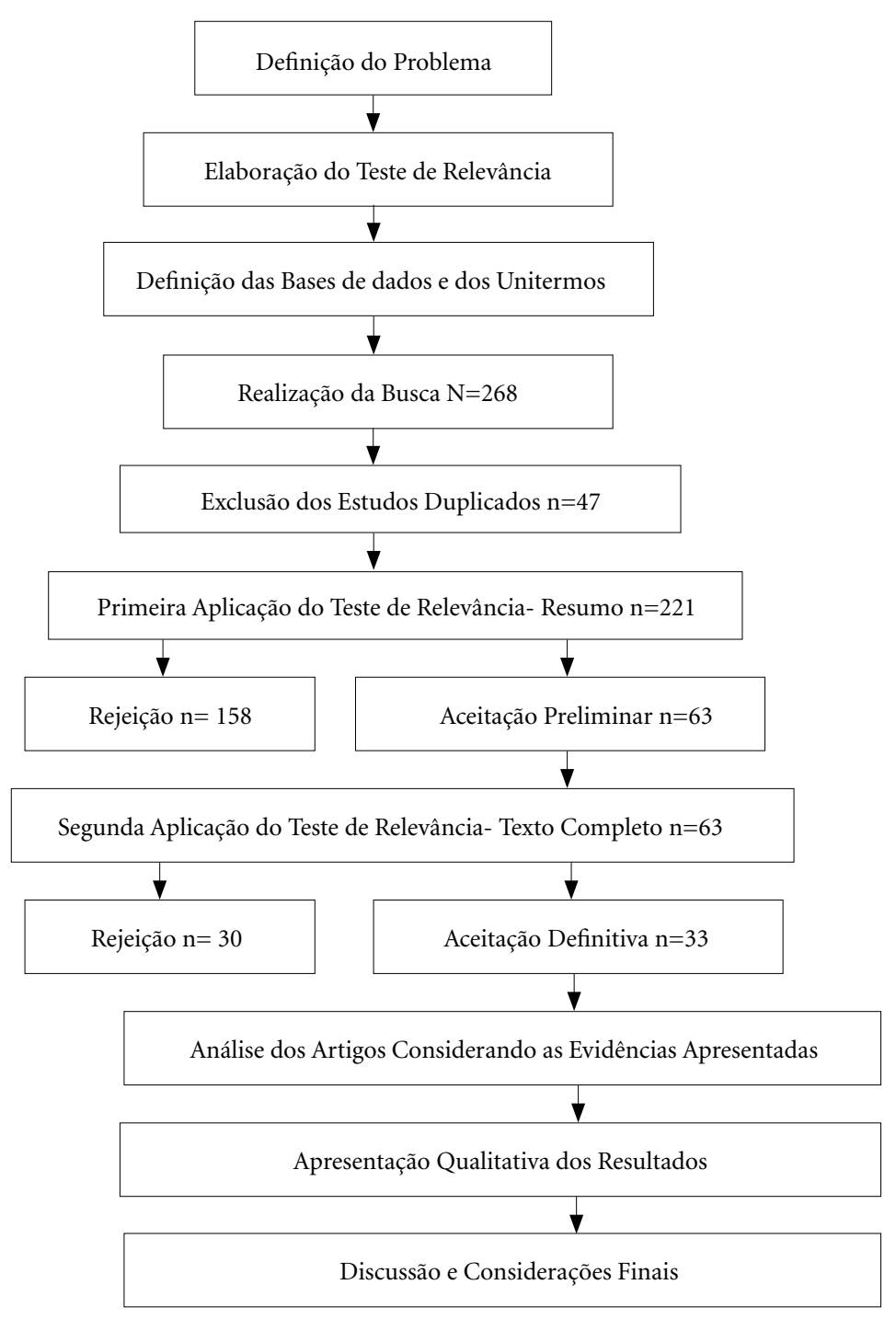

Figura 1. Diagrama de fluxo para a condução de uma Revisão Sistemática de Literatura (RSL).

Fonte: Adaptado de $\mathrm{Olsen}^{17}$.

Após a segunda aplicação do TR nos artigos completos, restaram 33 estudos que foram analisados qualitativamente no presente estudo.

\section{Resultados}

Fizeram parte desta revisão 33 estudos, destes, 09 (27,3\%) foram realizados no Brasil;

04 (12,1\%) nos Estados Unidos da América; $03(9,1 \%)$ no Reino Unido; $02(6,1 \%)$ na Nova Zelândia e na Sérvia; e 01 (3,0\%) para os demais países: Índia, México, Qatar, Kwait, África, Sué- cia, Costa Rica, Irlanda, Colômbia, Portugal, Nigéria, Austrália e Venezuela.

No que se refere aos motivos de estoque: medicamentos estocados/possível utilização no futuro teve destaque, sendo citado em 12 (36,4\%) dos artigos; a aquisição sem prescrição médica ou automedicação em 9 (27,3\%) artigos. Quanto ao descarte, $22(66,7 \%)$ dos artigos apontaram que a forma mais frequente é o descarte no lixo comum; seguido por descarte em rede de esgoto 21 (63,6\%) (Quadro 1).

As evidências encontradas nos artigos foram organizadas em quadro sinóptico, de acordo 
Quadro 1. Sinopse dos estudos que abordam os motivos de estoque e formas de descarte de medicamentos no domicílio.

\begin{tabular}{|c|c|c|c|}
\hline Autores (Ano) Periódico & \begin{tabular}{|c|} 
Localização \\
geográfica
\end{tabular} & Motivos de estoque & Formas de Descarte \\
\hline $\begin{array}{l}\text { Bound; Voulvoulis }{ }^{19}(2005) \\
\text { Environ Health }\end{array}$ & \begin{tabular}{|l|} 
Reino \\
Unido \\
\end{tabular} & $\begin{array}{l}\text { Não abordou estoque de } \\
\text { medicamentos. }\end{array}$ & $\begin{array}{l}\text { Lixo doméstico; esgoto; farmácias/ } \\
\text { estabelecimentos de saúde. }\end{array}$ \\
\hline $\begin{array}{l}\text { Fanhani et al. }{ }^{20}(2006) \\
\text { Arq Cienc Saúde UNIPAR }\end{array}$ & Brasil & $\begin{array}{l}\text { Automedicação; } \\
\text { medicamentos estocados. }\end{array}$ & Não abordou a forma de descarte. \\
\hline $\begin{array}{l}\text { Ekedahl }{ }^{21}(2006) \\
\text { Pharm World Sci }\end{array}$ & Suécia & $\begin{array}{l}\text { Medicamento vencido; } \\
\text { óbito; sobra; melhora clínica; } \\
\text { mudança no tratamento. }\end{array}$ & Não abordou a forma de descarte. \\
\hline $\begin{array}{l}\text { Jiménez et al. }{ }^{22}(2006) \\
\text { Rev Costarric Salud } \\
\text { Pública }\end{array}$ & Costa Rica & $\begin{array}{l}\text { Utilização em caso de } \\
\text { emergência, falta de adesão ao } \\
\text { tratamento. }\end{array}$ & $\begin{array}{l}\text { Lixo comum; esgoto; disposição no } \\
\text { solo; farmácias/estabelecimentos de } \\
\text { saúde }\end{array}$ \\
\hline $\begin{array}{l}\text { Seehusen e Edwards }{ }^{23} \\
(2006) \\
\text { J Am Board Fam Med }\end{array}$ & $\begin{array}{l}\text { Estados } \\
\text { Unidos da } \\
\text { América }\end{array}$ & $\begin{array}{l}\text { Não abordou estoque de } \\
\text { medicamentos. }\end{array}$ & $\begin{array}{l}\text { Lixo doméstico; esgoto; farmácia/ } \\
\text { estabelecimentos de saúde. }\end{array}$ \\
\hline $\begin{array}{l}\text { Abahussain et al. }{ }^{24}(2006) \\
\text { Med Princ Pract }\end{array}$ & Kuwait & $\begin{array}{l}\text { Mudanças ou abandono do } \\
\text { tratamento. }\end{array}$ & $\begin{array}{l}\text { Lixo doméstico; esgoto; farmácias/ } \\
\text { estabelecimentos de saúde. }\end{array}$ \\
\hline $\begin{array}{l}\text { Bound et al. }{ }^{25}(2006) \\
\text { Environ Toxicol Phar }\end{array}$ & $\begin{array}{l}\text { Reino } \\
\text { Unido }\end{array}$ & Guardam para uso futuro. & $\begin{array}{l}\text { Solo; esgoto; farmácias/ } \\
\text { estabelecimento de saúde e doações. }\end{array}$ \\
\hline $\begin{array}{l}\text { Musson et al. }{ }^{26}(2007) \\
\text { J Air Waste Manag Assoc }\end{array}$ & $\begin{array}{l}\text { Reino } \\
\text { Unido }\end{array}$ & $\begin{array}{l}\text { Não abordou estoque de } \\
\text { medicamentos. }\end{array}$ & $\begin{array}{l}\text { Lixo doméstico; esgoto; farmácias/ } \\
\text { estabelecimentos de saúde. }\end{array}$ \\
\hline $\begin{array}{l}\text { Margonato et al. }{ }^{27}(2008) \\
\text { Cad Saúde Pública }\end{array}$ & Brasil & $\begin{array}{l}\text { Aquisição } \\
\text { excessiva/ Automedicação. }\end{array}$ & Não abordou a forma de descarte. \\
\hline $\begin{array}{l}\text { Bueno et al. }{ }^{28}(2009) \\
\text { Rev Ciênc Farm Básica Apl }\end{array}$ & Brasil & $\begin{array}{l}\text { Utilização no futuro; doação; } \\
\text { automedicação; estoque de } \\
\text { medicamentos. }\end{array}$ & $\begin{array}{l}\text { Lixo doméstico; esgoto; disposição } \\
\text { no solo; farmácias/estabelecimento } \\
\text { de saúde. }\end{array}$ \\
\hline $\begin{array}{l}\text { Braund et al. }{ }^{29}(2009) \\
\text { Pharm World Sci }\end{array}$ & $\begin{array}{l}\text { Nova } \\
\text { Zelândia }\end{array}$ & $\begin{array}{l}\text { Mudanças no tratamento; } \\
\text { falta de adesão ao tratamento. }\end{array}$ & Não abordou a forma de descarte. \\
\hline $\begin{array}{l}\text { James et } \text { al. }^{30}(2009) \\
\text { Ann Pharmacother }\end{array}$ & $\begin{array}{l}\text { Nova } \\
\text { Zelândia }\end{array}$ & $\begin{array}{l}\text { Oferta excessiva; } \\
\text { mudanças no tratamento; } \\
\text { falta de adesão; óbito; sobra } \\
\text { de medicamentos vencidos. }\end{array}$ & Não abordou a forma de descarte. \\
\hline $\begin{array}{l}\text { Kotchen et al. }{ }^{31}(2009) \mathrm{J} \\
\text { Environ Manag }\end{array}$ & $\begin{array}{l}\text { Estados } \\
\text { Unidos da } \\
\text { América }\end{array}$ & $\begin{array}{l}\text { Não abordou estoque de } \\
\text { medicamentos. }\end{array}$ & $\begin{array}{l}\text { Lixo doméstico; esgoto; farmácias/ } \\
\text { estabelecimentos de saúde. }\end{array}$ \\
\hline $\begin{array}{l}\text { Gupta et al. } .^{32}(2011) \\
\text { Int J Pharm Sci Res }\end{array}$ & Índia & $\begin{array}{l}\text { Não abordou o motivo de } \\
\text { estoque. }\end{array}$ & Lixo doméstico; esgoto. \\
\hline $\begin{array}{l}\text { Kheir et al. }{ }^{33}(2011) \\
\text { Drug Healthc Patient Saf }\end{array}$ & Qatar & $\begin{array}{l}\text { Não abordou estoque de } \\
\text { medicamentos. }\end{array}$ & $\begin{array}{l}\text { Lixo doméstico; esgoto; farmácias/ } \\
\text { estabelecimentos de saúde. }\end{array}$ \\
\hline $\begin{array}{l}\text { Sasu et al. }{ }^{34}(2012) \\
\text { Waste Manag Res }\end{array}$ & África & Utilização no futuro. & $\begin{array}{l}\text { Lixo doméstico; esgoto; } \\
\text { descaracterização por aquecimento; } \\
\text { farmácias e estabelecimentos de saúde. }\end{array}$ \\
\hline $\begin{array}{l}\text { Beckhauser et al. }{ }^{35}(2012) \\
\text { Rev Ciênc Farm Básica Apl }\end{array}$ & Brasil & $\begin{array}{l}\text { Sobra de tratamentos } \\
\text { anteriores. }\end{array}$ & Não abordou a forma de descarte. \\
\hline $\begin{array}{l}\text { Laste et al. }{ }^{36}(2012) \\
\text { Ciênc Saúde Colet }\end{array}$ & Brasil & $\begin{array}{l}\text { Automedicação e } \\
\text { medicamentos estocados. }\end{array}$ & Não abordou a forma de descarte. \\
\hline $\begin{array}{l}\text { Kusturica et al. }{ }^{37}(2012) \\
\text { Int. J. Clin Pharm. }\end{array}$ & Sérvia & $\begin{array}{l}\text { Não abordou estoque de } \\
\text { medicamentos. }\end{array}$ & $\begin{array}{l}\text { Lixo doméstico; esgoto; } \\
\text { descaracterização por aquecimento; } \\
\text { farmácia e estabelecimentos de saúde. }\end{array}$ \\
\hline $\begin{array}{l}\text { Wieczorkiewicz et al. }{ }^{38} \\
\text { (2013) Ann. Pharmacother. }\end{array}$ & Unidos & $\begin{array}{l}\text { Não abordou o motivo de } \\
\text { estoque. }\end{array}$ & $\begin{array}{l}\text { Lixo doméstico; esgoto; farmácia e } \\
\text { estabelecimento de saúde. }\end{array}$ \\
\hline $\begin{array}{l}\text { Iob et al. } .^{39}(2013) \\
\text { Infarma - Ciências Farm. }\end{array}$ & Brasil & $\begin{array}{l}\text { Sobra de medicamentos e } \\
\text { utilização no futuro. }\end{array}$ & $\begin{array}{l}\text { Lixo doméstico; esgoto; por } \\
\text { aquecimento; farmácia e } \\
\text { estabelecimentos de saúde. }\end{array}$ \\
\hline
\end{tabular}


Quadro 1. Sinopse dos estudos que abordam os motivos de estoque e formas de descarte de medicamentos no domicílio.

\begin{tabular}{|c|c|c|c|}
\hline Autores (Ano) Periódico & $\begin{array}{c}\text { Localização } \\
\text { geográfica }\end{array}$ & Motivos de estoque & Formas de Descarte \\
\hline $\begin{array}{l}\text { Vellinga et al. }{ }^{40}(2014) \text { Sci. } \\
\text { Total Environ. }\end{array}$ & Irlanda & $\begin{array}{l}\text { Utilização no futuro; } \\
\text { desconhecimento sobre o } \\
\text { descarte; doação. }\end{array}$ & $\begin{array}{l}\text { Lixo doméstico; esgoto; farmácias e } \\
\text { estabelecimento de saúde. }\end{array}$ \\
\hline $\begin{array}{l}\text { Lehardt et al. }{ }^{41}(2014) \\
\text { UNOPAR Cient Ciênc } \\
\text { Biol Saúde. }\end{array}$ & Brasil & Utilização no futuro. & $\begin{array}{l}\text { Lixo doméstico; esgoto; farmácias e } \\
\text { estabelecimento de saúde. }\end{array}$ \\
\hline $\begin{array}{l}\text { Gracia-Vásquez et al. }{ }^{42} \\
(2014) \\
\text { Int J Clin Pharm }\end{array}$ & México & $\begin{array}{l}\text { Prescrição excessiva; } \\
\text { automedicação; amostras } \\
\text { grátis; cessação, mudança; não } \\
\text { adesão ao tratamento; óbito. }\end{array}$ & Lixo doméstico; rede de esgoto. \\
\hline $\begin{array}{l}\text { Pinto et al. }{ }^{43}(2014) \text { Eng. } \\
\text { Sanit. Ambient. }\end{array}$ & Brasil & $\begin{array}{l}\text { Não abordou o motivo de } \\
\text { estoque. }\end{array}$ & $\begin{array}{l}\text { Lixo doméstico; esgoto; farmácia e } \\
\text { estabelecimentos de saúde; resíduos } \\
\text { recicláveis. }\end{array}$ \\
\hline $\begin{array}{l}\text { Lystlund et al. }{ }^{44}(2014) \mathrm{J} \\
\text { Am Pharm Assoc. }\end{array}$ & $\begin{array}{l}\text { Estados } \\
\text { Unidos da } \\
\text { América }\end{array}$ & $\begin{array}{l}\text { Melhora clínica; mudança } \\
\text { no tratamento; amostra } \\
\text { grátis. }\end{array}$ & $\begin{array}{l}\text { Lixo doméstico; esgoto; disposição } \\
\text { no solo. }\end{array}$ \\
\hline $\begin{array}{l}\text { kusturica et al. }{ }^{45}(2015) \\
\text { Cent. Eur. J. Public Health }\end{array}$ & Sérvia & Sobra e automedicação. & Não abordou a forma de descarte. \\
\hline $\begin{array}{l}\text { Piveta et al. }{ }^{46}(2015) \\
\text { Semina: Ciências } \\
\text { Biológicas e da Saúde }\end{array}$ & Brasil & Utilização futura & $\begin{array}{l}\text { Lixo doméstico; locais específicos de } \\
\text { recolhimento e esgoto. }\end{array}$ \\
\hline $\begin{array}{l}\text { Bergen et al. }{ }^{47}(2015) \\
\text { Australian Prescriber }\end{array}$ & Austrália & $\begin{array}{l}\text { Óbito, mudança no } \\
\text { tratamento, preocupação com } \\
\text { a segurança e eficácia. }\end{array}$ & $\begin{array}{l}\text { Descarte adequado: Programa } \\
\text { Nacional de Retorno e Eliminação } \\
\text { de Medicamentos Indesejados. }\end{array}$ \\
\hline $\begin{array}{l}\text { Quijano-Prieto et al. }{ }^{48} \\
(2016) \\
\text { Rev Salud Pública }\end{array}$ & Colômbia & $\begin{array}{l}\text { Sobra: melhora clínica e } \\
\text { suspensão do tratamento. }\end{array}$ & $\begin{array}{l}\text { No lixo doméstico e descarte nos } \\
\text { postos de coleta. }\end{array}$ \\
\hline $\begin{array}{l}\text { Dias-Ferreira et al. }{ }^{49} \\
(2016) \\
\text { Waste Manag Res }\end{array}$ & Portugal & $\begin{array}{l}\text { Utilização futura, para } \\
\text { emergência; não vencidos e } \\
\text { prescrição excessiva. }\end{array}$ & $\begin{array}{l}\text { Farmácia; estabelecimentos de } \\
\text { saúde; lixo doméstico; esgoto e } \\
\text { resíduos recicláveis. }\end{array}$ \\
\hline $\begin{array}{l}\text { Banwat et al. } .^{0}(2016) \\
\text { Trop J Pharm Res }\end{array}$ & Nigeria & $\begin{array}{l}\text { Automedicação; sobra e } \\
\text { utilização futura. }\end{array}$ & $\begin{array}{l}\text { Lixo doméstico; rede de esgoto e } \\
\text { queimavam. }\end{array}$ \\
\hline $\begin{array}{l}\text { Correia; Marcano }{ }^{51}(2016) \\
\text { Rev Int Contam Ambie }\end{array}$ & Venezuela & $\begin{array}{l}\text { Automedicação; sobra e } \\
\text { utilização futura. }\end{array}$ & Lixo doméstico; rede de esgoto. \\
\hline
\end{tabular}

com: autores, ano, periódico, localização geográfica, motivos de estoque e formas de descarte (Quadro 1).

\section{Discussão}

\section{Estoque de medicamentos no domicílio}

Os estudos analisados indicam que são diversos os motivos que levam a população a estocar medicamentos no domicílio. Dentre eles destacam-se a possível utilização do medicamento no futuro, aquisição sem prescrição médica, alteração no tratamento ou mudança na dosagem, sobra de tratamentos anteriores, óbito do paciente, falta de adesão ou abandono do tratamento, excesso de oferta e prescrição na dose excessiva, recebimento de amostras grátis e possibilidade de doação para outras pessoas $^{20-22,24,27-30,34-36,39,40,42,44,46-51}$.

Dentre os fatores que propiciam o estoque de medicamentos, a automedicação e a aquisição de medicamentos sem prescrição trazem os principais riscos para a população, considerando a toxicidade potencial de alguns medicamentos $^{26,41,43,51}$. Estas atitudes refletem uma cultura persistente em distintas regiões do mundo com a qual se banalizam os riscos derivados do uso inadequado de algum princípio ativo e/ou das 
reações adversas associadas à ingestão de determinados medicamentos ${ }^{39}$.

A venda de medicamentos em volume superior ao especificado pela prescrição médica, é também um indutor para o estoque de medicamentos nos domicílios. Nesse sentido, as estratégias que estimulem a venda fracionada de medicamentos respeitando a dose indicada são necessárias, posto que diminuem a geração e o acúmulo de resíduos e a contaminação do meio ambiente $13,35,39,43,52-54$.

Outros fatores determinantes são os erros de prescrição e dispensação ou mudanças sucessivas dos tratamentos, que obrigam o paciente a adquirir medicamentos em excesso e sentir necessidade de guardá-los fora de uso, em virtude do custo econômico do mesmo, com a finalidade de conseguir aproveitar no futuro ou doar a outras pessoas, evitando dessa forma maior dispêndio de dinheiro.

As condições socioeconômicas também favorecem a compra e o estoque de medicamentos, dentre eles cabe destacar as intensas campanhas publicitárias na mídia que apontam resultados terapêuticos excessivamente favoráveis e criam expectativas na população perante a proposta de cura e alívio ${ }^{39}$. A distribuição de amostras grátis, fornecidas pelos laboratórios farmacêuticos também representam fatores preponderantes na manutenção de pequenas farmácias domiciliares $^{13,43,52,55-59}$. Esta realidade do estoque domiciliar de medicamentos é um problema de saúde pública que deve ser desestimulado para minimizar possíveis agravos na população ${ }^{20,22,27,32,35-40,44,60}$.

Ações educativas voltadas para desestimular o estoque domiciliar de medicamentos, a automedicação e o fracionamento na dose prescrita são de extrema importância para a prevenção de intoxicações medicamentosas ${ }^{39}$. Nesse contexto, acredita-se que a formação de profissionais de saúde capacitados para a orientação correta sobre a utilização e armazenamento de medicamentos, assim como para estimular a adesão aos tratamentos possam ser medidas que diminuam o acúmulo dos medicamentos no domicílio $^{21,23,25,27,34,36,40,44}$.

\section{Descarte de medicamentos no domicílio}

Os estudos elencados nesta revisão apontam como principais formas de descarte dos medicamentos nos domicílios: lixo comum, redes de esgoto, a devolução para os estabelecimentos de saúde, a disposição no solo, e descaracterização por fogo ${ }^{19,22-26,28,31-34,37-44,46-51}$.
A gestão do descarte de medicamentos é um desafio mundial. Diariamente toneladas de resíduos são coletadas e em sua maioria gerenciadas, inadequadamente, ocasionando efeitos indesejáveis e irreversíveis ao meio ambiente $\mathrm{e}^{16,51,52,59,61}$. O descarte de medicamentos nas redes de esgoto e a disposição no solo, mediante o encaminhamento junto aos resíduos urbanos, representam uma ameaça para a saúde humana, integridade ambiental e biodiversidade do planeta ${ }^{1,25,33,41,49,51,62}$.

A presença de fármacos nos solos e nas águas, destacando-se os antibióticos, estrogênios, antineoplásicos e os imunossupressores, podem gerar subprodutos potencialmente tóxicos de difícil decomposição, com efeitos teratogênicos, mutagênicos e carcinogênicos nas populações animais e humanas ${ }^{19,22,35,37,38-40}$. A descaracterização por fogo, procedimento comum nos domicílios rurais, também representa riscos para a saúde humana e ambiental devido à emissão de gases tóxicos poluentes ${ }^{37}$.

$\mathrm{Na}$ tentativa de diminuir o impacto ambiental provocado pelo descarte inadequado de resíduos no Brasil, a Anvisa por meio da RDC No 222 de 2018, regulamentou as boas práticas de Gerenciamento dos Resíduos de Serviços de Saúde (RSS) e determinou que resíduos de fármacos descartados por serviços de saúde, farmácias, drogarias, distribuidores de medicamentos ou apreendidos, necessitam ser expostos a tratamento ou ser desprezados em aterro de resíduos perigosos - Classe I. ${ }^{63}$

No entanto, não há uma política pública nacional que regulamente a coleta e o descarte domiciliar de RSS. A regulamentação acontece de forma isolada em alguns municípios e estados, como por exemplo: o Rio Grande de Sul (nas cidades de Passo Fundo e Porto Alegre), Amazonas, Paraíba, Mato Grosso (no município de Cuiabá), Acre e o Paraná, ou por meio de ações educativas e estratégias específicas, como o Programa Descarte Consciente Compartilhado entre empresas, consumidores e órgãos públicos, onde são

determinados postos de coleta para a população descartar seus medicamentos ${ }^{64-66}$.

No cenário internacional, os estudos demonstraram alguns programas de recolhimento e descarte correto de medicamentos, tais como: "Take-back Program" desenvolvidos e executados na Nova Zelândia, Gana, Estados Unidos, Irlanda, e Suécia. Esses programas visam orientar a comunidade quanto a importância de realizar a disposição ambientalmente adequada dos medicamentos presentes nos domicílios, assim como estimular o retorno dos medicamentos 
vencidos e/ou que não estão sendo utiliza$\operatorname{dos}^{21,26,30,34,44}$. Apenas a Austrália adotou uma política pública de forma gratuita quanto ao descarte adequado dos medicamentos em nível nacional, denominado National Return and Disposal of Unwanted Medicines (NATURUM), o qual foi instituído em 1998 e está disponível em todas as farmácias ${ }^{47}$.

Os achados revelaram que a simples instalação de postos de coleta de medicamentos ou programas de recolhimento, não têm se mostrado eficientes sem a orientação à população que gere mudanças de percepção e de hábitos no dia-adia $^{1,25,39,43,49}$. A população necessita de educação em saúde, que permita o reconhecimento das práticas corretas para a guarda e para o descarte de medicamentos. Práticas que favoreçam rotas de descarte e descaracterização de medicamentos ecologicamente adequadas devem ser estimuladas na população, considerando

não só as comunidades de hoje, mas também as gerações futuras ${ }^{25,41,50}$.

\section{Considerações finais}

Os estudos analisados demonstram que o estoque de medicamentos no domicílio é uma prática comum nos diversos países, podendo facilitar a prática da automedicação. No que se refere às formas de descarte dos medicamentos, estas apresentam riscos para a saúde pública, tendo em vista que as principais vias para desprezo dos medicamentos ainda são o lixo comum e as redes de esgoto. Neste sentido se faz necessário o incentivo para a elaboração/implementação de políticas públicas voltadas para o uso e o descarte adequado de medicamentos no domicílio.

É fundamental a ampliação de programas de educação em saúde como ferramenta para conscientização da população sobre as práticas corretas de armazenamento e descarte dos medicamentos no domicílio, tendo em vista o desafio que essas questões representam para a saúde humana e ambiental.

\section{Colaboradores}

VM Constantino, BM Fregonesi, KAA Tonani, GS Zagui, APC Toninato, ERS Nonose, LA Fabriz e SI Segura-Muñoz participaram em todas as etapas e de forma relevante no desenho e desenvolvimento do texto. 


\section{Referências}

1. Lunardelli A, Machado ID, Monteiro SC. Programa de descarte apropriado do rejeito medicamentoso como ferramenta institucional educacional. Rev Eletr Farm 2017; 14(1):32-38.

2. World Health Organization (WHO). Essential medicines and health products. Geneva: WHO; 2017. [acessado 2017 Ago 14]. Disponível em: http://www.who. int/medicines/areas/rational_us e/ en /

3. Centers for Disease Control Prevention (CDC). Antibiotic Prescribing and Use. 2018. [acessado $2018 \mathrm{Mar}$ 25]. Disponível em: https://www.cdc.gov/antibioticuse/index.html

4. Organização Pan-Americana de Saúde (OPAS). Uso racional de medicamentos: fundamentação em condutas terapêuticas e nos macroprocessos da assistência farmacêutica. Brasília: Organização Pan-americana de Saúde/Organização Mundial de Saúde; 2016. (boletim informativo) 1(12).

5. Yousif MA. In-home drug storage and utilization habits: a Sudanese study. East Mediterr Health J 2002; 8(2-3):422-431.

6. Brasil. Ministério da Saúde (MS). Uso racional de medicamentos. Brasília: MS; 2014. [acessado 2017 Mar 27]. Disponível em: http://portal.saude.gov.br/portal/ saude/area.cfm?id_area $=1141$

7. Brasil. Ministério da Saúde (MS). Política Nacional de Medicamentos. Brasília: MS; 2001.

8. Agência Nacional de Vigilância Sanitária (Anvisa). Resolução RDC no 20, de 5 de maio de 2011. Dispõe sobre o controle de medicamentos à base de substâncias classificadas como antimicrobianos, de uso sob prescrição, isoladas ou em associação. 2011. [acessado 2018 Abr 08]. Disponível em: http://bvsms.saude.gov. br/bvs/saudelegis/anvisa/2011/rdc0020_05_05_2011. html

9. Agência Nacional de Vigilância Sanitária (Anvisa). Resolução da Diretoria Colegiada - RDC no 68, de 28 de novembro de 2014. Dispõe sobre a atualização do Anexo I, Lista de Antimicrobianos Registrados na Anvisa, da Resolução - RDC no 20, de 5 de maio de 2011 e dá outras providências. 2014. [acessado $2018 \mathrm{Abr}$ 08]. Disponível em: http://www.poderesaude.com.br/ novosite/images/publicacoes_01.12.2014-II.pdf

10. Falqueto E, Kligerman DC, Assumpção RF. Como realizar o correto descarte de resíduos de medicamentos? Cienc Saude Colet 2010; 15(2):3283-3293.

11. Brasil. Lei no 12.305 , de 2 de agosto de 2010. Institui a Política Nacional de Resíduos Sólidos; altera a Lei n ${ }^{\circ}$ 9.605 de 12 de fevereiro de 1998 e dá outras providências. Diário Oficial da União 2010; 3 ago.

12. Agência Nacional de Vigilância Sanitária (Anvisa). A Anvisa e a implantação da logística reversa de resíduos de medicamentos. Brasília: Anvisa; 2014. [acessado 2017 Jul 15]. Disponível em: http://portal.anvisa.gov. $\mathrm{br} / \mathrm{wps} / \mathrm{wcm} / \mathrm{connect}$

13. Barros JF, Egea JS. Descarte de medicamentos e sustentabilidade: uma abordagem importante da produção ao uso. Encontro Nacional da Anppas [periódico na internet]. 2012. Belém - PA. [acessado 2017 Maio 13]. Disponível em: http://www.anppas.org.br/encontro6/anais/ARQUIVOS/GT4-868-623.pdf
14. Pinto EA. Impacto ambiental dos medicamentos [dissertação]. Porto: Universidade Fernando Pessoa; 2011.

15. Balbino EC, Balbino MLC. O descarte de medicamentos no Brasil: Um olhar socioeconômico e ambiental do lixo farmacêutico. Revista Âmbito Jurídico [periódico na internet]. [acessado 2017 Jul 15]. Disponível em: http://www.ambito-juridico.com.br/site/index. php? artigo_id=9187\&n_link=revista_artigos_leitura

16. Mazzer C, Cavalcanti OA. Introdução à gestão ambiental de resíduos. Infarma Ciênc Farmac 2004; 16:11-12.

17. Olsen J. Meta-analysis or collaborative studies. JOEM 1995; 8:897-902.

18. Ferreira PC, Piai KA, Takayanagui AMM, Segura-Muñoz SI. Aluminum as a risk fator for Alzheimer's dissecasse. Rev Latino-Am Enfermagem 2008; 16(1):151-157.

19. Bound JP, Voulvoulis N. Household disposal of pharmaceuticals as a pathway for aquatic contamination in the United Kingdom. Environ Health 2005; 113(12):1705-1711.

20. Fanhani HR, Correa MI, Lourenço EB, Fernandes ED, Billó VL, Lorenson L, Spiguel PKS, Galoro JLF, Takemura OS, Andrade OG. Avaliação domiciliar da utilização de medicamentos por moradores do jardim Tarumã, município de Umuarama - PR. Arq Cienc Saúde da Unipar 2006; 10(3):127-131.

21. Ekedahl ABE. Reasons why medicines are returned to Swedish pharmacies unused. Pharm World Sci 2006; 28:352-358.

22. Jiménez H, Guillermo L, Sáenz RB. Medicamentos subutilizados em el ámbito comunitario, área salud coronado, Costa Rica. Rev Costarric Salud Pública 2006; 15(28):50-61.

23. Seehusen DA, Edwards J. Patient Practices and Beliefs Concerning Disposal of Medications. J Am Board Fam Med 2006; 19(6):542-547.

24. Abahussain EA, Ball DE, Matowe WC. Practice and opinion towards disposal of unused medication in Kuwait. Med Princ Pract 2006; 15:352-357.

25. Bound JP, Kitsou K, Voulvoulis N. Household disposal of pharmaceuticals and perception of risk to the environment. Environ Toxicol Phar 2006; 21(3):301-307.

26. Musson SE, Townsend T, Seaburg K, Mosa J. A continuous collection system for household pharmaceutical wastes: a pilot project. J Air Waste Manag Assoc 2007; 57(7):828-835.

27. Margonato FB, Thomson Z, Paoliello MMB. Determinantes nas intoxicações medicamentosas agudas na zona urbana de um município do Sul do Brasil. Cad Saude Publica 2008; 24(2):333-341.

28. Bueno CS, Weber D, Oliveira KR. Farmácia caseira e descarte de medicamentos no bairro Luiz Fogliatto do município de Ijuí - RS. Rev Ciênc Farm Básica Apl 2009; 30(2):201-210.

29. Braund R, Gn G, Matthews R. Investigating unused medications in New Zealand. Pharm World Sci 2009; 31:664-669.

30. James TH, Helms ML, Braund R. Analysis of medications returned to community pharmacies. Ann Pharmacother 2009; 43:1631-1635. 
31. Kotchen M, Callao's J, Wheeler K, Wong C, Zahller M. Pharmaceuticals in wastewater: Behavior, preferences, and willingness to pay for a disposal program. J Environ Manag 2009; 90:1476-1482.

32. Gupta J, Alam N, Bhardwaj A, Amin F. Prospective survey study on assessment and education of home medicine cabinet in general population of community. Int J Pharm Sci Res 2011; 2(5):1237-1243.

33. Kheir N, Hajj MSE, Wilbur K, Kaissi RML, Yousif A. An exploratory study on medications in Qatar homes. Drug Healthc Patient Saf 2011; 3:99-106.

34. Sasu S, Kümmerer K, Kranert M. Assessment of pharmaceutical waste management at selected hospitals and homes in Ghana. Waste Manag Res 2012; 30(6):625-630.

35. Beckhauser GC, Valgas C, Galato D. Perfil do estoque domiciliar de medicamentos em residências com crianças. Rev Ciênc Farm Básica Apl 2012; 33(4):583589.

36. Laste G, Deitos A, Kauffmann C, Castro LC, Torres IS, Fernandes LC. Papel do agente comunitário de saúde no controle do estoque domiciliar de medicamentos em comunidades atendidas pela Estratégia de Saúde da Família. Cienc Saude Colet 2012; 17(5):1305-1312.

37. Kusturica MP, Sabo A, Tomic Z, Horvat O, Solak Z. Storage and disposal of unused medications: knowledge, behavior, and attitudes among Serbian people. Int J Clin Pharm 2012; 34:604-610.

38. Wieczorkiewicz SM, Kassamali Z, Danziger LH. Behind closed doors: medication storage and disposal in the home. Ann Pharmacother 2013; 47(4):482-489.

39. Iob GA. Análise da forma de descarte de medicamentos por usuários de uma unidade de saúde no município de Porto Alegre. Infarma Ciênc Farm 2013; 25(3):118-125.

40. Vellinga A, Cormican S, Driscoll J, Furey, M, O’sullivan $\mathrm{M}$, Cormican M. Public practice regarding disposal of unused medicines in Ireland. Sci Total Environ 2014; 478:98-102.

41. Lenhardt EH, Sólis LJB, Cintra EVCS, Botelho EHL. O descarte de medicamentos no bairro Grande Terceiro, Cuiabá-MT. UNOPAR Cient Ciênc Biol Saúde 2014; 16(1):5-8.

42. Gracia-Vásquez SL, Ramírez-Lara E, Camacho-Mora IA, Cantú-Cárdenas LG, GraciaVásquez YA, EsquivelFerriño PC, Ramírez-Cabrera MA, Gonzalez-Barranco P. An analysis of unused and expired medications in Mexican households. Int J Clin Pharm 2015; 37(1):121-126.

43. Pinto GMF, Silva KR, Pereira RFAB, Sampaio SI. Estudo do descarte residencial de medicamentos vencidos na região de Paulínia (SP), Brasil. Rev Eng Sanit Ambient 2014; 19(3):219-224.

44. Lystlund S, Stevens E, Planas LG, Marcy T. Patient participation in a clinic-based community pharmacy medication take-back program. J Am Pharm Assoc 2014; 54:280-284.

45. Kusturica MP, Tomic Z, Horvat O, Pavlovi N, Mikov M. Antibiotics in Serbian Households: a source of potencial health and environmental threats? Cent Eur J Public Health 2015; 23(2):114-118.
46. Piveta LN, Silva LB, Guidoni CM, Girotto E. Armazenamento e descarte de medicamentos por acadêmicos da área da saúde de uma universidade pública paranaense. Semina: Ciências Biológicas e da Saúde 2015; 36(1):55-66.

47. Bergen PJ, Hussainy SY, George J, Kong DCM, Kirkpatrick CMJ. Safe dispo sal of prescribed medicines. Australian Prescriber 2015; 38(3):90-92.

48. Quijano-Prieto D, Orozco-Díaz JG, Holguín-Hernández E. Patients' knowledge and practices about unconsumed drugs disposal. An Approach to Ecopharmacovigilance. Rev Salud Publica 2016; 18(1):61-71.

49. Dias-Ferreira C, Valente S, Vaz J. Practices of pharmaceutical waste generation and discarding in households across Portugal. Waste Manag Res 2016; 34(10):1006-1013.

50. Banwat SB, Auta A, Dayom DW, Buba Z. Assessment of the storage and disposal of medicines in some homes in Jos north local government area of Plateau State, Nigeria. Trop J Pharm Res 2016 May; 15(5):989.

51. Correia A, Marcano L. Evaluación de las rutas de entrada de competes farmacêuticos de uso doméstico al ambiente caso estudio: município Valencia, Estado Carabobo, Venezuela. Rev. Int. Contam Ambie 2016; 32(1):77-86.

52. Barcelos MN, Peres AP, Pereira IO, Chavasco LSI, Freitas DF. Aplicação do método FMEA na identificação de impactos ambientais causados pelo descarte doméstico de medicamentos. Eng Ambient 2011; 8(4):62-68.

53. Falqueto E, Kligerman DC. Diretrizes para um programa de recolhimento de medicamentos vencidos no Brasil. Cienc Saude Colet 2013; 18(3):883-892.

54. Falqueto E, Kligerman DC. Análise normativa para descarte de resíduos de medicamentos - estudo de caso da região sudeste do Brasil. Rev de Direito Sanitário 2012; 13(2):10-23.

55. Alvarenga LSV, Nicoletti MA. Descarte doméstico de medicamentos e algumas considerações sobre o impacto ambiental decorrente. Revista Saúde 2010; 4(3):34-39.

56. Frigieri MC, Gasparini AR, Gasparini JC. Estudo do descarte de medicamentos e consciência ambiental no município de Catanduva-SP. Ciência \& Tecnologia: FATEC Jaboticabal 2011; 2(1):38-51.

57. João WSJ. Descarte de medicamentos. Pharmacia Brasileira 2011; 82:14-16.

58. Nakazone MA, Araújo GLB, Pinheiro A, Takano C, Godoy MF, Souza DRS. Quantificação do desperdício de medicamentos na prática clínica. Arq Cienc Saúde 2006; 13(2):75-81.

59. Tope TRG, Araújo LEB. Contaminação do meio ambiente pelo descarte inadequado de medicamentos vencidos ou não utilizados. Hoppe \& Araújo 2012; 6(6):1248-1262.

60. Thach AV, Brown CM, Pope N. Consumer perceptions about a community pharmacy-based medication take back program. J Environ Manag 2013; 127:23-27.

61. Silva SP, Lins AMSC, Santos RS. A relevância da educação ambiental no processo de gerenciamento dos resíduos sólidos decorrentes do tratamento do câncer. Enciclopédia Biosfera 2012; 8(15):2367. 
62. Abahussain EA, Ball DE. Disposal of unwanted medicines from households in Kuwait. Pharm World Sci 2007; 29:368-373.

63. Agência Nacional de Vigilância Sanitária (Anvisa). Resolução RDC nº 222, de 28 de março de 2018. Regulamenta as boas práticas de gerenciamentos dos resíduos de serviços de saúde e dá outras providências. 2018. [acessado 2018 Abr 08]. Disponível em: http://portal. imprensanacional.gov.br/web/guest/consulta?p_p_id

64. Brasil Health Service (BHS). Programa Descarte Consciente. 2014. [acessado 2017 Ago 07]. Disponível em: http://www.descarteconsciente.com.br

65. Cruz MJB, Azevedo AB, Cruz HL, Bodevan EC, Araújo LU, Santos DF. Descarte de medicamentos em municípios do Vale do Jequitinhonha, Minas Gerais, Brasil. Vigil Sanit Debate 2017; 5(1):84-90.

66. Paraná. Decreto no 9.213, de 23 de outubro de 2013. Regulamenta a Lei no 17.211, de 03 de julho de 2012, que dispõe sobre a responsabilidade da destinação dos medicamentos em desuso no Estado do Paraná e seus procedimentos e dá providências. 2013. [acessado 2018 Mar 20]. Disponível em: https://www.legisweb. com.br/legislacao/?id=261098

Artigo apresentado em 20/12/2017

Aprovado em 18/06/2018

Versão final apresentada em 20/06/2018 